\title{
Label-free characterization of Amyloid- $\beta$-plaques and associated lipids in brain tissues using stimulated Raman scattering microscopy
}

\author{
Volker Schweikhard ${ }^{1 *}$, Andrea Baral ${ }^{2}$, Vishnu Krishnamachari ${ }^{1}$, William C. Hay ${ }^{1}$, Martin Fuhrmann ${ }^{2}$ \\ ${ }^{1}$ Leica Microsystems CMS GmbH, Am Friedensplatz 3, 68165 Mannheim, Germany \\ ${ }^{2}$ Neuroimmunology and Imaging Group, German Center for Neurodegenerative Diseases (DZNE), Bonn, 53127, Germany. \\ *all correspondence should be addressed to volker.schweikhard@leica-microsystems.com
}

\begin{abstract}
:
The brains of patients with neurodegenerative diseases such as Alzheimer's Disease (AD) often exhibit pathological alterations that involve abnormal aggregations of proteins and lipids. Here, we demonstrate that high-resolution, label-free, chemically-specific imaging using Stimulated Raman Scattering Microscopy (SRS) provides novel insights into the biophysical properties and biochemical composition of such pathological structures. In brain slices of a mouse model of AD, SRS reveals large numbers of Amyloid- $\beta$ plaques that commonly form a characteristic, three-dimensional core-shell structure, with a fibrillar proteinaceous core surrounded by a halo-like shell of lipid-rich deposits. SRS spectroscopic imaging allows for a clean, label-free visualization of the misfolded ( $\beta$-sheet) Amyloid- $\beta$ content in the plaque core. Surrounding lipid-rich deposits are found to contain comparatively high concentrations of membrane lipids (sphingomyelin, phosphatidylcholine), but lower levels of cholesterol than healthy white matter structures. Overall, the SRS spectra of plaque-associated lipids closely resemble those of nearby neurites, with the notable difference of a higher degree of lipid unsaturation compared to healthy brain structures. We hypothesize that plaque-associated lipid deposits may result from neuritic dystrophy associated with $A D$, and that the observed increased levels of unsaturation could help identify the kinds of pathological alterations taking place. Taken together, our results highlight the potential of Stimulated Raman Scattering microscopy to contribute to a deeper understanding of neurodegenerative diseases.
\end{abstract}




\section{Introduction}

Alzheimer's Disease (AD) is a chronic neurodegenerative disease that is characterized by neurological symptoms such as progressive cognitive impairment and altered behavior, accompanied by neuronal cell death and the buildup of senile plaques and other pathological changes in certain areas of the brain. And yet, despite decades of intensive research, the molecular and cell biological mechanisms of disease progression remain incompletely understood, and there is currently no efficient treatment to cure, halt, or slow down the progression of the disease. An important hallmark of AD pathology is the aggregation of misfolded Amyloid- $\beta(A \beta)$ peptides into large abnormal extracellular deposits. Furthermore, it is commonly believed that neurodegeneration in $A D$ is linked to the presence of small $A \beta$ oligomers in the intracellular space that interfere with neurotransmission. However, the aberrant formation, transport, and defective clearance of these neurotoxic $A \beta$ species in brain tissues remains incompletely understood. Several hypotheses suggest that plaque-associated lipids may act as surfactants that mediate an exchange of $A \beta$ between aggregated forms that are sequestered in the extracellular plaques and soluble small oligomeric forms that lead to toxicity. However, the origins and roles of these plaqueassociated lipids in the progression of the disease remain poorly understood. As a consequence, there is a high demand for new analytical methods that can probe the interplay between brain lipids and $A \beta$ with high spatial resolution and chemical specificity in intact brain tissues.

Coherent Raman Scattering (CRS) microscopy denotes a set of nonlinear optical microscopy techniques, most prominently Coherent Anti-Stokes Raman Scattering (CARS) and Stimulated Raman Scattering (SRS), that image biological structures by exploiting the characteristic, vibrational contrast of the sample molecules ${ }^{1,2,3}$. The optical setup and principle of SRS microscopy are illustrated in

Supplemental Figure $\mathbf{S 1}$ and described in Materials and Methods. In recent years, CRS has emerged as a powerful approach for label-free, biochemical imaging of neuronal structures and for dynamic monitoring of functional parameters such as membrane potential ${ }^{4}$ and neurotransmitter concentrations ${ }^{5}$. In addition, CRS has been used in fundamental research on neurodegenerative diseases, to characterize Alzheimer's pathology ${ }^{6,7,8}$, Parkinson's Disease ${ }^{9}$ and multiple sclerosis ${ }^{10}$, or to study peripheral nerve degeneration in ALS ${ }^{11}$. Currently, there is a major push to develop label-free histopathological analyses based on CRS (sometimes integrated in a multi-modal approach with other nonlinear optical imaging techniques such as two-photon fluorescence or harmonic-generation microscopy) $)^{12}$, with major efforts in brain cancer ${ }^{13,14,15}$, other cancers ${ }^{16,17,18}$ and a range of other diseases ${ }^{19,20}$. Beyond disease-diagnosis, SRS has been applied in fundamental research on disease mechanisms, e.g. to link aberrant cholesterol metabolism pathways to prostate cancer aggressiveness ${ }^{16}$ and to identify lipid desaturation as a marker for cancer stem cells ${ }^{17}$.

Here, we demonstrate the potential of SRS to provide label-free, high-resolution biochemical and biophysical characterizations of healthy and diseased brain tissues. Our results provide novel insights into Amyloid- $\beta$ plaque biology and highlight the potential of coherent Raman scattering microscopy to contribute to a deeper understanding of neurodegenerative diseases. 


\section{Results}

\section{Label-free SRS microscopy of diseased brain slices reveals lipid-rich pathological deposits associated with $A \beta$ plaques}

We applied SRS microscopy to unstained brain slices from mice carrying an amyloid precursor protein and a presenilin 1 mutation that exhibit AD-like pathology ${ }^{21,22}$, as well as from healthy control mice. We first acquired large-area overview tile-scan SRS images of healthy and diseased brain slices (Figure 1 (A) and (B), respectively). In these images, SRS contrast arises from $\mathrm{CH}_{2}$ stretch vibrations at $2850 \mathrm{~cm}^{-1}$ predominantly found in lipids. Lipid-rich white matter regions (bright horizontal bands at image bottom) are easily discernible, as well as the less lipid-rich grey matter of the cortex (darker green regions in the top half of the images). In diseased brain slices, additional, localized lipid-rich pathological structures are seen, which are absent in the healthy cortex. Further detailed analysis described below demonstrates that these localized pathological structures correspond to lipid deposits associated with Amyloid- $\beta$ plaques. Figure 1 (C) shows a high-resolution two-color SRS image of such an Amyloid- $\beta$ plaque (red, SRS at $1678 \mathrm{~cm}^{-1}$ as explained below), and lipid structures (green, SRS at $2850 \mathrm{~cm}^{-1}$ ). Plaques are found to exhibit a characteristic, three-dimensional core-shell structure, in which a fibrillar core is surrounded by a halo-like arrangement of lipid-rich structures. Orthogonal slice views through a 3D image stack, and a movie of the stack, are shown in Supplemental Figure S2 and Supplemental Movie S1, respectively. In the healthy tissue regions surrounding the plaque, SRS reveals myelinated neurites (bright tubular structures) and neuronal cell bodies (dark regions devoid of lipids) embedded in homogenous grey matter. The observation of prominent plaque-associated lipid deposits raises questions: What is the source of these lipids, and how do these structures form? What is their role in AD progression? In the following, we present a detailed characterization of $A \beta$ plaques and associated lipids, utilizing the quantitative spectroscopic imaging capabilities of SRS, to begin to address these questions.
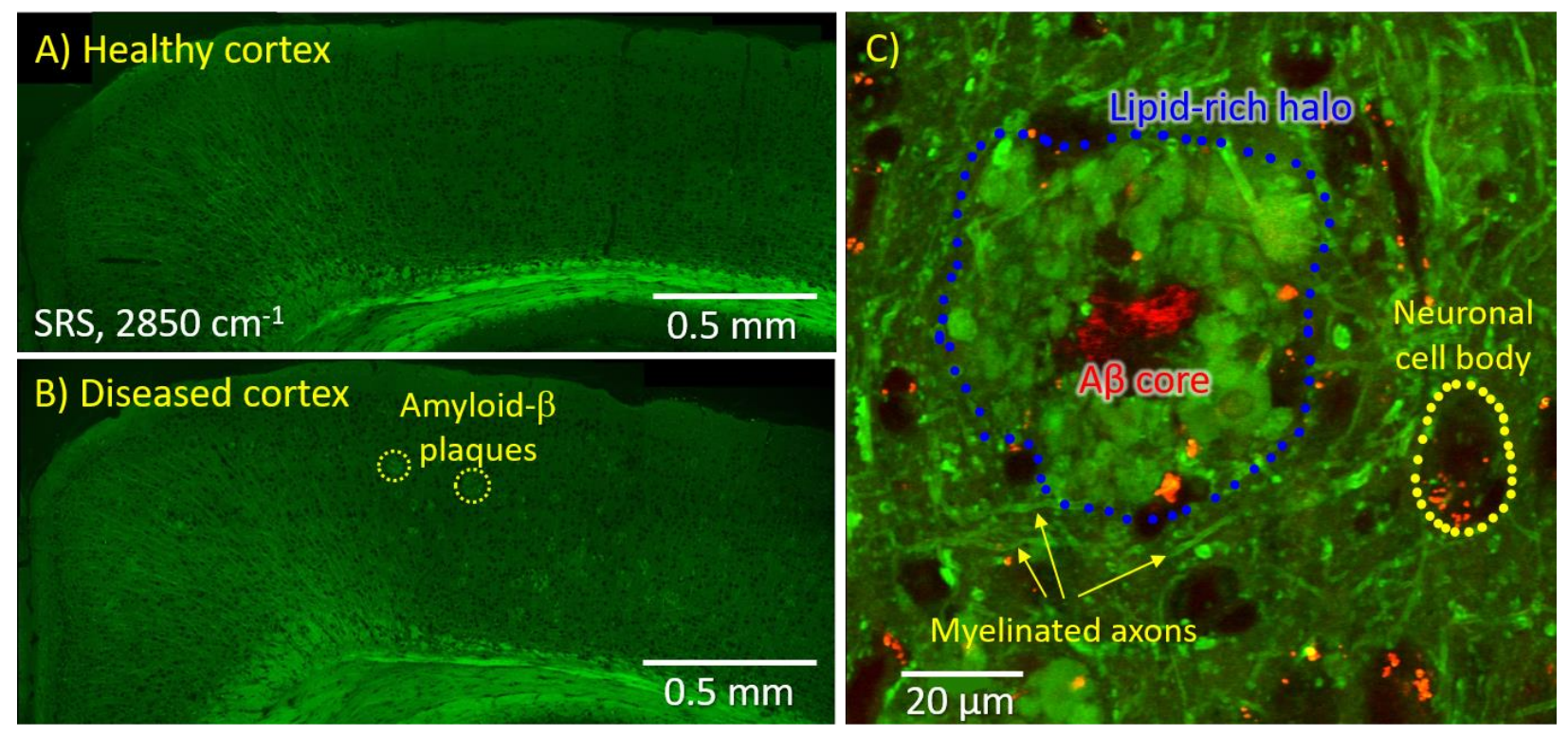

Figure 1: (A, B) Label-free, large-area overview images of unlabeled mouse brain slices, acquired using Stimulated Raman Scattering microscopy (SRS). Signals arising from $\mathrm{CH}_{2}$ stretch vibrations at $2850 \mathrm{~cm}^{-1}$ predominantly found in lipids are shown in a healthy (A) and a diseased (B) mouse cortex. (C) High-resolution, two-color SRS image of an Amyloid- $\beta$ plaque (red, SRS at $1678 \mathrm{~cm}^{-1}$ ), and associated lipid deposits (green, SRS at $2850 \mathrm{~cm}^{-1}$ ). 


\section{High-resolution hyperspectral SRS microscopy of healthy and diseased brain structures}

To characterize the biochemical composition of Amyloid- $\beta$ plaques, we performed hyperspectral SRS microscopy, in which SRS images are acquired sequentially over a large range of wavenumbers. As a result, a full SRS spectrum with a spectral resolution of $\sim 12 \mathrm{~cm}^{-1}$ is available for each image pixel. Importantly, it has been shown that the resulting SRS spectra are fully equivalent to spontaneous Raman spectra and therefore allow for similar image analysis and quantification procedures developed for spontaneous Raman data. CARS spectra, by contrast, suffer from spectral distortions and off-resonant background signals that make a quantification considerably more difficult.

Representative example images in Figure $\mathbf{2}(\mathbf{A}, \mathbf{C})$ show a tissue region at the interface between the cortex (bottom of image) and white matter (top). Representative spectra of white matter, grey matter, cell nuclei, $A \beta$ plaque cores and plaque-associated lipid halo regions are shown in Figure $\mathbf{2}$ (B). Note, the spectral data shown in Figure $\mathbf{2}$ are raw data that did not require any post-processing (i.e., no background subtraction and no smoothing), and were acquired on regular fused silica coverslips. This is a major advantage of coherent Raman imaging, in which the signal is generated selectively in the laser focus, over spontaneous Raman imaging, which suffers from large spectral backgrounds originating from substrates and/or sample mounting compounds, and hence require extensive data subtraction procedures and the use of specialized, expensive substrates that produce lower background signals $\left(\mathrm{MgF}_{2}, \mathrm{CaF}_{2}\right)$.

SRS spectra comprise two regions, (i) the high-wavenumber region ( $2800-3100 \mathrm{~cm}^{-1}$ ) with signals from $\mathrm{CH}_{2}$ and $\mathrm{CH}_{3}$ stretch vibrations representing the sample's total protein and lipid contents (2940, $2850 \mathrm{~cm}^{-1}$, respectively), and $=\mathrm{C}-\mathrm{H}$ vibrations associated with unsaturated lipids $\left(\sim 3020 \mathrm{~cm}^{-1}\right)$; and (ii) the fingerprint region ( $500-1800 \mathrm{~cm}^{-1}$ ) containing large numbers of peaks that are often unique for a given compound.

Notable features in the fingerprint spectra of brain slices include (i) the near-absence of $\mathrm{C}=\mathrm{O}$ stretch signal from oxidized lipids (esters) around $1740 \mathrm{~cm}^{-1}$, (ii) a prominent peak around $1660 \mathrm{~cm}^{-1}$ comprising the Amide I $\mathrm{C}=\mathrm{O}$ vibration of proteins and $\mathrm{C}=\mathrm{C}$ stretch vibrations in unsaturated lipids and sterols. (iii) a prominent double peak around $1465 \mathrm{~cm}^{-1}$ (Amide II in proteins) and $1445 \mathrm{~cm}^{-1}$ (total lipid signal from $\mathrm{CH}_{2}$ bending), (iv) $1200-1400 \mathrm{~cm}^{-1}$ region comprising the Amide III mode from proteins and further lipid signatures $\left(1300,1268 \mathrm{~cm}^{-1}\right)$, (v) 1020-1150 $\mathrm{cm}^{-1}$ signals from carbohydrates, (vi) a sharp peak at $1005 \mathrm{~cm}^{-1}$ from Tryptophan, (vii) peaks around 850 and $900-950 \mathrm{~cm}^{-1}$ mainly from carbohydrates and nucleic acids, and (viii) a region from $600-800 \mathrm{~cm}^{-1}$ containing weak but distinct signals that allow the distinction of several major lipid classes (Cholesterol at $707 \mathrm{~cm}^{-1}$, membrane lipids - mainly phosphatidylcholine (PC) and sphingomyelin (SM) at $723 \mathrm{~cm}^{-1}$, as well as phosphatidylethanolamine (PE) around $\left.760 \mathrm{~cm}^{-1}\right)$. Note, SRS spectra are highly reproducible between different brain slices imaged on different days, as demonstrated in Supplemental Figure S3, and therefore even small variations in the spectra can contain highly relevant information. In the following, we examined the biochemical and biophysical information contained in several of these features in more detail. 


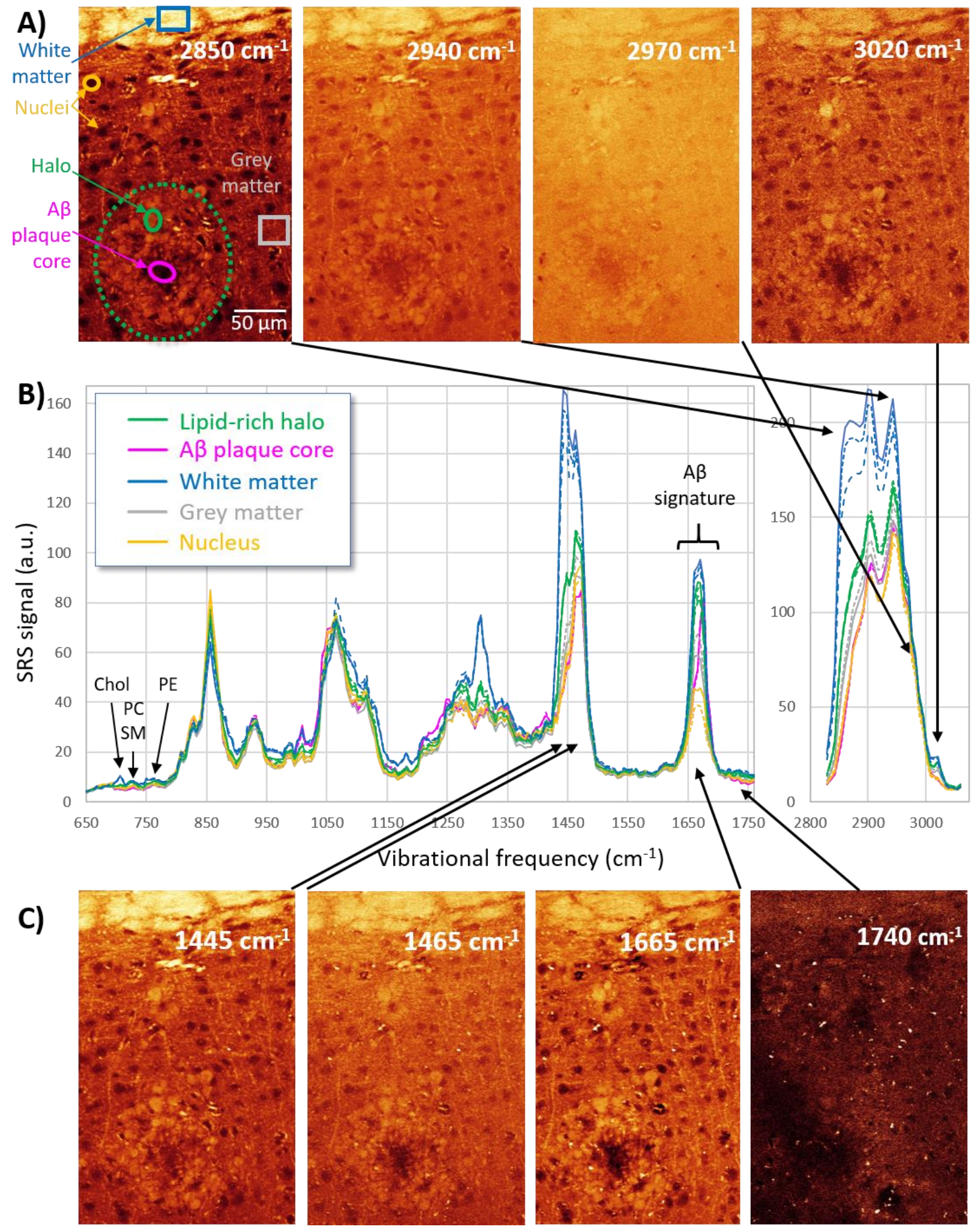

Figure 2: Hyperspectral SRS microscopy applied to a diseased mouse cortex. SRS images are acquired sequentially at a range of wavenumbers, as in the representative images shown in $(A, C)$. As a result, a full SRS spectrum is available for each image pixel. Representative SRS spectra of the brain structures indicated in (A) are shown in (B). 2-3 spectra from different instances of the same structure are shown to indicate typical spectral variation. For detailed explanations and analysis of the "A $\beta$-signature", see Figure 3. For "Chol, PC/SM, PE", see Figure 4. 


\section{SRS allows for a clean, label-free visualization of A $\beta$-peptide aggregates localized in the plaque cores.}

We first sought to identify a way of directly and specifically visualizing the aggregated $A \beta$ content of the pathological structures. We focused on the Amide I ( $C=O$ stretch) vibration, which was shown to be particularly sensitive to protein secondary structure in previous spontaneous Raman measurements ${ }^{23,24}$ and coherent Raman imaging studies ${ }^{6,7}$.

In Figure 3, we present a hyperspectral SRS analysis of the Amide I spectral region. Images taken at $1665 \mathrm{~cm}^{-1}$ show the overall tissue architecture, with contrast contributed both from the Amide I mode of proteins and from the overlapping $\mathrm{C}=\mathrm{C}$ stretch vibration of unsaturated lipids. These images display a notable absence of signal in the central "core" of the pathological structure that is enclosed by the lipid deposits. When tuning the SRS contrast to $1675 \mathrm{~cm}^{-1}$, bright fibrillar structures appear in this location, whereas contrast in the remainder of the tissue is somewhat weakened. We assign these fibrillar structures and their blue-shifted Amide-I mode to Amyloid- $\beta$ aggregates. This assignment agrees with a characteristic $\sim 10 \mathrm{~cm}^{-1}$ spectral blue-shift of the Amide I mode for proteins folded into a $\beta$-sheet conformation that was found in previous spontaneous Raman measurements on AD-affected tissues ${ }^{23,24}$. We validated the Amyloid- $\beta$ specificity of the $1675 \mathrm{~cm}^{-1}$ peak by co-localization with fluorescent signals from Methoxy-04 (MX04), a molecule that binds to peptides in $\beta$-sheet conformation and is commonly used to stain $A \beta$ plaques in Alzheimer's research (see Supplemental Figure S4). Our assignment also agrees with results from two previous studies on $A D$-affected tissues ${ }^{6,7}$, where similar structures exhibiting the same frequency-shift were observed and validated as $A \beta$ plaques using fluorescent dye labeling with thioflavin $S$, another stain commonly used to visualize $A \beta$.

The ratio of images at taken at $1675 \mathrm{~cm}^{-1}$ and $1665 \mathrm{~cm}^{-1}$, shown in Figure 3 (A) (iii), therefore provides a clean and specific visualization of $A \beta$ aggregates in plaques on an otherwise homogeneous background. We note that cell nuclei are visible with a very weak contrast in this image as well. This effect is seen in healthy brain slices too, and should therefore not be interpreted as evidence for the presence of $A \beta$ in cell nuclei. Figure 3 (C) shows a line profile of the intensity ratio $I^{1675} / I^{1665}$ through the $A \beta$ plaque core and a nearby cell nucleus, demonstrating the high specificity of this intensity ratio for the $A \beta$ core. 

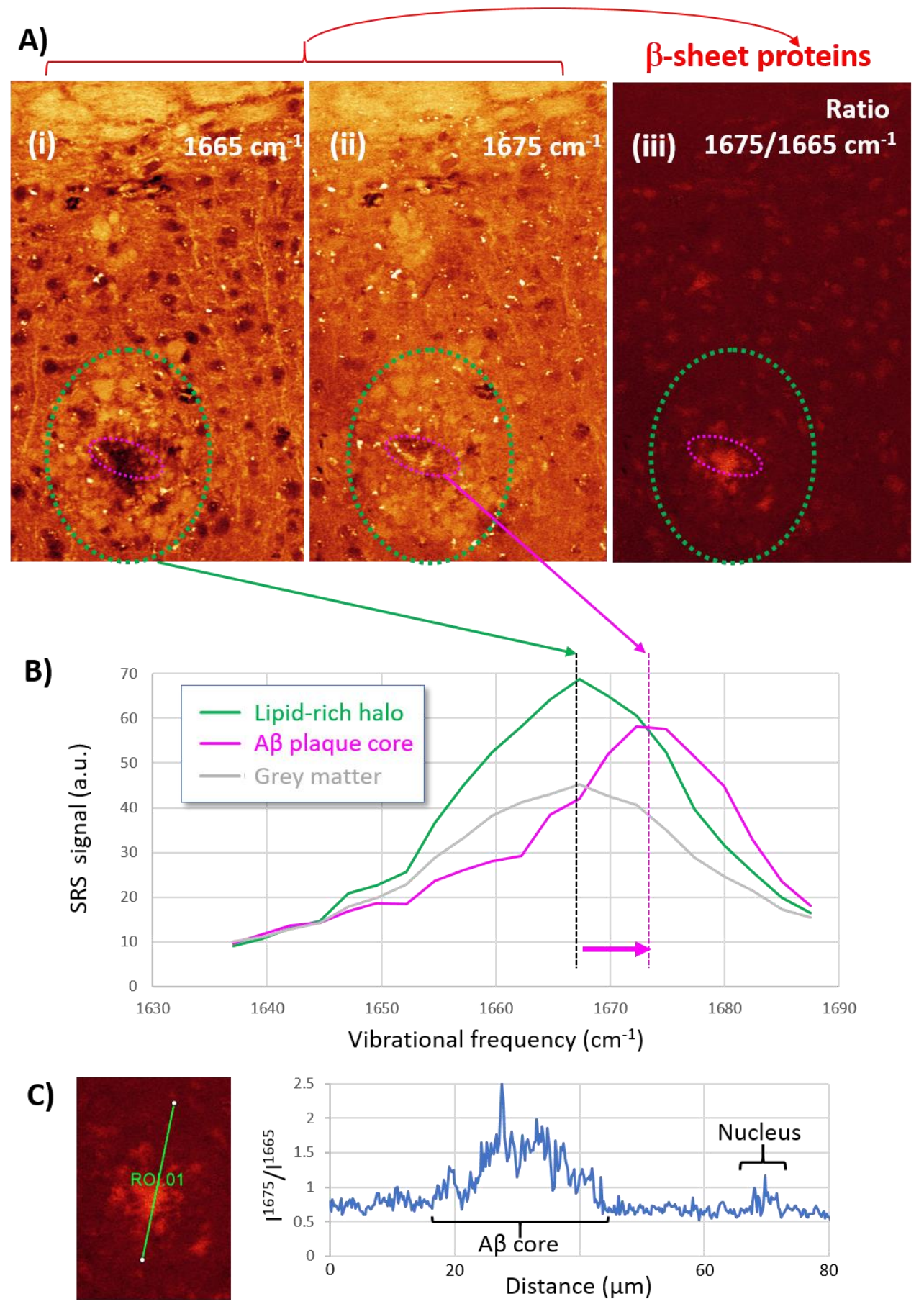

Figure 3: Hyperspectral SRS microscopy of the Amide I band reveals A $\beta$-aggregates in the plaque core. (A) (i) SRS images at $1665 \mathrm{~cm}^{-1}$ (Amide I vibration in regular proteins, as well as $\mathrm{C}=\mathrm{C}$ stretch in unsaturated lipids) exhibit a dark void in the core of the $A \beta$ plaque (pink line). (ii) At $1675 \mathrm{~cm}^{-1}$ (shifted Amide I vibration in $\beta$-sheet proteins), strong signals from fibrillar structures appear in the plaque core, consistent with the known misfolded $\beta$-sheet structure of $A \beta$-aggregates. (iii) Ratiometric visualization of $A \beta$ plaques. The ratio of intensities at 1675 and 1665 $\mathrm{cm}^{-1}$ is shown, providing a clean visualization of the plaque core. (B) SRS spectra of healthy grey matter, lipid-rich regions in the plaque halo and core region are shown, demonstrating the specific frequency shift of the Amide I mode in the plaque core compared to regular proteins in the surrounding grey matter. (C) Line profile of the intensity ratio $\mathrm{I}^{1675} / \mathrm{I}^{1665}$ through the $A \beta$ core and a nearby cell nucleus. 


\section{$A \beta$ plaque associated lipid deposits are rich in membrane lipids, but low in cholesterol}

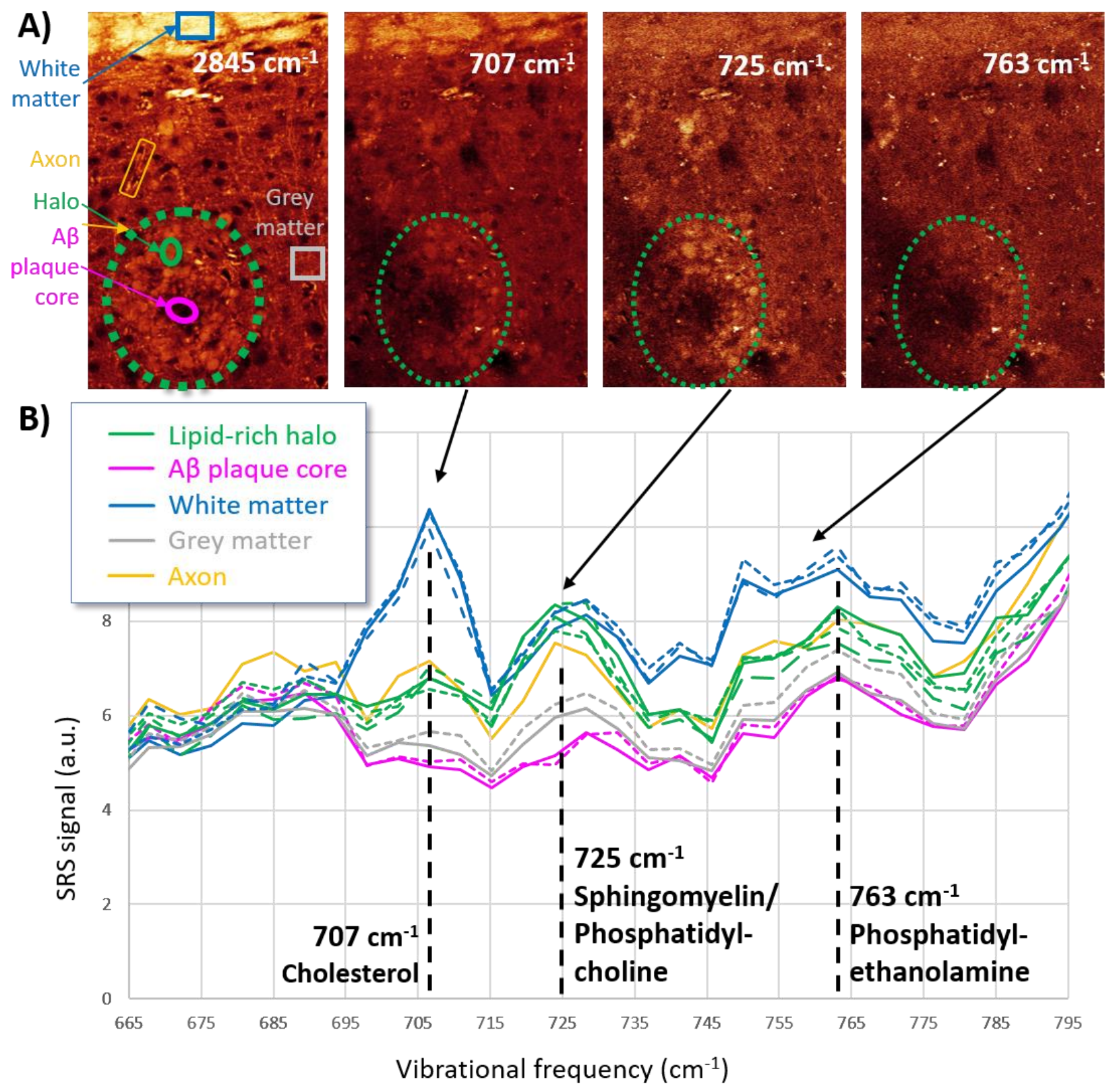

Figure 4: Stimulated Raman Scattering microscopy probes the composition of plaque-associated lipids and healthy brain structures. SRS images (A) and spectra (B) of the sterol ring breathing mode $\left(707 \mathrm{~cm}^{-1}\right)$, choline group vibrations $\left(725 \mathrm{~cm}^{-1}\right)$ and the ethanolamine group vibration from phosphatidylethanolamine $\left(763 \mathrm{~cm}^{-1}\right)$ are shown. An image of the total lipid content acquired at $2845 \mathrm{~cm}^{-1}$ is shown for reference in the first panel.

The lipidome of the healthy brain is known to be composed largely of glycerophospholipids, sphingolipids, and cholesterol, which localize predominantly to neuronal membranes and myelin ${ }^{25}$. However, several contradicting hypotheses exist as to the origin of plaque-associated lipids in $A D$. We therefore decided to investigate the spectral range from $700-770 \mathrm{~cm}^{-1}$ in detail, which contains characteristic fingerprint signatures for some of the major lipid classes ${ }^{26}$ (Figure 7). The sterol ring breathing mode from cholesterol is found at $707 \mathrm{~cm}^{-1}$. While images at this wavenumber show that plaque halos (image area encircled in 
green) do contain some cholesterol, it is present there at substantially lower levels than in white matter regions at the top edge of the image. By contrast, plaques are comparatively rich in membrane lipids (sphingomyelin SM and phosphatidylcholine $\mathrm{PC}, 725 \mathrm{~cm}^{-1}$ ). A signature from phosphatidylethanolamine $(P E)$ is seen in the spectra as well (feature around $745-775 \mathrm{~cm}^{-1}$ ). Consistent with published spectra ${ }^{26}$, the PE peak at $760 \mathrm{~cm}^{-1}$ is substantially broader than the ones of cholesterol and SM/PC. Images at this wavenumber suggest that $P E$ is more diffusely distributed throughout the entire tissue region, with the highest PE concentrations found in white matter, and a notable exclusion of PE in the plaque core. Note, while the spectral signatures from cholesterol, PC, SM and PE in the $695-775 \mathrm{~cm}^{-1}$ region investigated here are very weak compared to the other signals examined in this work, all spectral features are highly reproducibly observed in very similar ratios in multiple different brain slices imaged on different days, as shown in Supplemental Figure S5.

Overall, plaque-associated lipids (green spectra) display an almost identical spectral signature to nearby neurites (yellow spectrum) in the spectral region shown in Figure 4, but differ significantly from the spectra of cholesterol-rich white matter (See also Supplemental Figure $\mathbf{S 6}$ for the full spectral range). Taken together, our observations suggest that membrane components from nearby neurites, rather than cholesterol-rich white matter pools, appear to be the likely source of plaque-associated lipids.

\section{Plaque-associated lipids exhibit higher degrees of unsaturation than nearby white matter}

Two further pathologically relevant biochemical metrics accessible with SRS are the degrees of lipid unsaturation and oxidation in tissues. In pure lipid samples, the ratio of $\mathrm{C}=\mathrm{C}$ stretch signal at $\sim 1665 \mathrm{~cm}^{-1}$ to $\mathrm{CH}_{2}$ bending signal at $\sim 1445 \mathrm{~cm}^{-1}$ has been widely accepted as a quantitative metric for the degree of lipid unsaturation. Three independent calibrations, presented by three different research groups ${ }^{26,27,28}$ are in agreement that the average number of $\mathrm{C}=\mathrm{C}$ bonds per lipid molecule $\mathrm{N}_{(\mathrm{C}=c)}$ is linearly related to the intensity ratio $\mathrm{I}^{1665} / \mathrm{I}^{1445}=0.60 * \mathrm{~N}_{(\mathrm{C}=\mathrm{c})}$. In samples containing proteins in addition to lipids, some care has to be taken before attempting a ratiometric quantification of lipid unsaturation, since the $1665 \mathrm{~cm}^{-1}$ peak also contains signals from Amide I protein vibrations, and the $1445 \mathrm{~cm}^{-1}$ lipid peak overlaps in part with the Amide II peak from proteins at $1465 \mathrm{~cm}^{-1}$. Figure 5 (A) shows data from brain slices in this spectral region. In the brain, a relatively clean spatial separation into lipid-rich structures and proteindominated grey-matter tissue provides a way of eliminating protein signals from SRS spectra, by subtracting the spectra of nearby grey-matter regions from those of more lipid-rich structures. The result of this subtraction is shown in Figure $\mathbf{5}$ (B) and Supplemental Figure S6, and represents spectra of the lipid component of these structures with relatively little remaining contamination from protein signals. Quantification of the degree of unsaturation in different brain structures then yields $N_{(c=c)}=$ 1.55(19) in plaque halos (average and standard error of 23 halo regions), much higher than in nearby white matter where $\mathrm{N}_{(\mathrm{C}=\mathrm{c})}=0.66(6)$ (average over 3 large regions), and in nearby unaffected axons where $\mathrm{N}_{(\mathrm{C}=\mathrm{C})}=0.90(45)$ (average over 6 axons). 

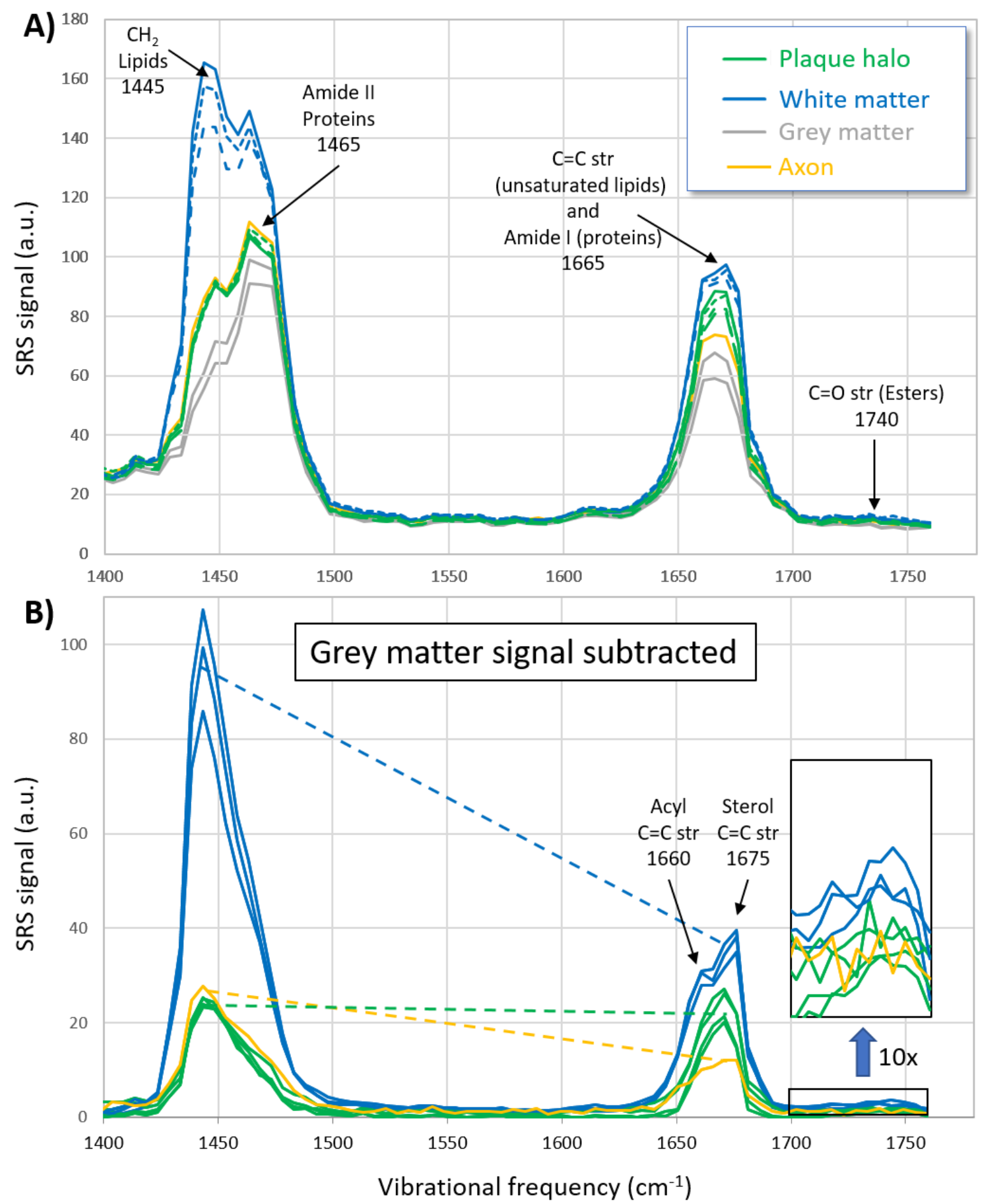

Figure 5: Ratiometric spectral analysis of lipid unsaturation. (A) Raw SRS spectra of the $\mathrm{CH}_{2}$ bend and $\mathrm{C}=\mathrm{C}$ stretch bands. (B) "Lipid-only" SRS spectra of brain structures, obtained by subtracting the spectra of nearby grey matter from the full spectra of white matter, plaque lipids, and axons. The ratio of SRS intensities at 1665 and $1445 \mathrm{~cm}^{-1}$ $\left(1^{1665} / I^{1445}\right)$ is directly proportional to the degree of lipid unsaturation. As indicated by the slope of the dashed lines, this ratio is higher in the plaque-halo compared to surrounding unaffected white matter and myelin-rich neurites. 
Note, a ratiometric analysis of unsaturation is possible using high-wavenumber spectra as well, (see Supplemental Figure S7) and supports the finding of higher unsaturation levels in plaque lipids compared to healthy structures. The ratio of intensities at $3020 \mathrm{~cm}^{-1}$ to $2850 \mathrm{~cm}^{-1}$ from grey-mattersubtracted high-wavenumber spectra yields $\mathrm{N}_{(\mathrm{C}=\mathrm{C})} \approx 1.6$ in plaque halos, $\mathrm{N}_{(\mathrm{C}=\mathrm{c})} \approx 0.5$ in white matter, and $\mathrm{N}_{(\mathrm{C}=\mathrm{C})} \approx 0.6$ in nearby axons. Note that signals from the $\mathrm{C}=\mathrm{C}$ bond in the sterol ring of cholesterol contribute to the $\mathrm{I}^{1665} / \mathrm{I}^{1445}$ measure of lipisd unsaturation, whereas cholesterol does not have a strong signal around $3020 \mathrm{~cm}^{-1}$; hence, the qualitative agreement of high-wavenumber and fingerprint metrics for unsaturation rules out a dominant role of the sterol $\mathrm{C}=\mathrm{C}$ vibration in our assessment of unsaturation.

An interesting feature of the "grey-matter-subtracted spectra" in Fig. 5 (B) is the splitting of the $\mathrm{C}=\mathrm{C}$ peak around $1670 \mathrm{~cm}^{-1}$ in white matter into a doublet $\left(1660\right.$ and $\left.1675 \mathrm{~cm}^{-1}\right)$. This splitting is explained by a spectral shift between $\mathrm{C}=\mathrm{C}$ vibrations in acyl and sterol groups ${ }^{29}$, both of which appear to be present in large concentrations in white matter. Note, the observed clear Cholesterol ring breathing signature in white matter at $707 \mathrm{~cm}^{-1}$ in Figure 4 and the strong sterol $\mathrm{C}=\mathrm{C}$ stretch in Figure 5, in combination with the near absence of $1740 \mathrm{~cm}^{-1}$ signal expected from cholesteryl esters, suggests that cholesterol is present in its unesterified form, consistent with prior observations in brain tissue ${ }^{30}$.

Interestingly, the near-absence of lipid $\mathrm{C}=\mathrm{O}$ stretch signals at $1740 \mathrm{~cm}^{-1}$ of plaque-associated lipids also demonstrates that oxidation levels remain low even in these pathological structures. This observation is somewhat surprising, since oxidative stress is a key feature in the Alzheimer's disease (AD) brain and has been found to manifest as lipid peroxidation ${ }^{31}$.

\section{Discussion}

Label-free SRS imaging of brain slices from a transgenic mouse model of Alzheimer's Disease reveals large numbers of $A \beta$ aggregates, which we find are consistently surrounded by a halo of lipid-rich deposits. Similar associations of lipids with $A \beta$ plaques have been observed in earlier studies using coherent Raman scattering ${ }^{6,7}$, imaging mass spectrometry ${ }^{32}$ and electron microscopy ${ }^{33}$, albeit with much lower spatial resolution or with a lack of chemical specificity. Interactions of lipids with pathological $A \beta$ structures are under active investigation for their relevance to $A D$ disease progression, because lipids are thought to have the capacity to extract small $A \beta$ oligomers from aggregates in a detergent-like fashion ${ }^{34}$, thereby facilitating their transport to the intracellular space where they become neurotoxic. In addition, several major lipid classes are known to be disrupted in AD, including cholesterol, sphingolipids, phospholipids, and glycerolipids ${ }^{25}$. And yet, despite their obvious importance, the precise roles of pathological lipid alterations in the progression of $A D$ have remained unclear.

Several hypotheses, not necessarily mutually exclusive, may explain the origin of plaqueassociated lipids: (i) the cholesterol hypothesis states that cholesterol may play a specific role in A $\beta$ plaque formation. This hypothesis is supported by the finding from mass spectrometric analyses that cholesterol and its transporter apoE are colocalized with $A \beta$ plaques in transgenic mouse models of $A D^{35}$. The mechanism by which cholesterol could be involved in $A D$ pathology is however still unclear ${ }^{25}$. (ii) the neuritic dystrophy hypothesis proposes that plaque-associated lipids may originate from the degeneration of neurites and myelin sheaths in $A D^{36}$. And, (iii) the immune system hypothesis suggests that plaque-associated lipids may originate from interactions of the brain's innate immune system with pathological $A \beta$ aggregates $37,38,39$. In particular, plaques have been found to be tightly enveloped by 
microglia, which were found to assemble a barrier with a strong impact on plaque composition and toxicity ${ }^{38}$. Insights into the validity of either one of these hypotheses could open new avenues of investigation into the roles of the lipids in disease progression, and could potentially lead to the identification of new therapeutic targets for AD.

Our findings on the composition of pathological lipid structures are most in line with the neuritic dystrophy hypothesis: A comparison of the full SRS spectra of plaque-associated lipids to those of healthy brain structures reveals a high degree of similarity to the spectra of nearby neurites. In particular, a strong SRS signature at $723 \mathrm{~cm}^{-1}$ suggests the presence of high concentrations of membrane lipids (phosphatidylcholine and sphingomyelin). By contrast, relatively weak signals from the sterol ring breathing mode at $707 \mathrm{~cm}^{-1}$ show that plaque-associated lipids contain less cholesterol than surrounding healthy white matter structures, disfavoring a dominant role of cholesterol metabolism and transport in the formation of these structures. A notable feature in the spectra of plaque-associated lipids is the high degree of unsaturation compared to those found in healthy brain structures: both, a ratiometric analysis of signals at $1665 \mathrm{~cm}^{-1}$ ( $\mathrm{C}=\mathrm{C}$ stretch, unsaturated lipids) and $1445 \mathrm{~cm}^{-1}\left(\mathrm{CH}_{2}\right.$ twist, total lipids), and the strength of the $3020 \mathrm{~cm}^{-1}=\mathrm{C}-\mathrm{H}$ stretch signal from unsaturated lipids compared to the total lipid signal at $2850 \mathrm{~cm}^{-1}\left(\mathrm{CH}_{2}\right.$ stretch) suggest that plaque-associated lipids exhibit higher degrees of unsaturation than white matter and neuritic structures, which themselves are already known to be very rich in (poly)unsaturated fatty acids. We hypothesize that these unique spectral signatures could help identify the kinds of pathological alterations taking place in neuritic structures as a consequence of AD.

\section{Conclusions}

We have applied a label-free chemical spectroscopic imaging approach based on Stimulated Raman Scattering microscopy to probe Amyloid- $\beta$ plaques in brain tissue slices from a transgenic mouse model of Alzheimer's Disease. Our results show that plaques consistently form a core-halo structure, with a dense proteinaceous $A \beta$ core surrounded by a halo of lipid-rich deposits. SRS enables a clean, label-free differentiation of the $A \beta$ core from the regular protein content of the surrounding unaffected brain tissue, by exploiting a spectral shift of the Amide I vibration that arises from the misfolding of aggregated $A \beta$ into a $\beta$-sheet conformation. A spectroscopic analysis of the surrounding plaqueassociated lipid deposits reveals that these contain high concentrations of the membrane lipids phosphatidylcholine and sphingomyelin. By contrast, the concentrations of cholesterol in plaques are low compared to those in nearby healthy lipid-rich brain structures. Overall, the spectroscopic similarity of plaque-associated lipids to nearby unaffected neurites favors the hypothesis that pathological lipid deposits form around the $A \beta$ core as a consequence of neuritic dystrophy processes associated with $A D$.

In the future, we anticipate that the spectroscopic imaging capabilities of SRS will enable similar, hypothesis-generating and -testing research in many areas of the life sciences and medicine $e^{40,41}$, ranging from fundamental research in cell and tissue biology, to preclinical research on disease mechanisms, to applications in histopathology and diagnostic imaging, and the identification of novel biomarkers for a wide range of diseases. The enormous information content of SRS spectroscopic imaging also highlights the potential of this technique as a versatile platform for label-free, high-content screening applications. 


\section{Materials and Methods}

\section{Transgenic mouse model:}

Mice that exhibit AD-like pathology were prepared as previously described ${ }^{21,22}$ : Wild-type mice were crossbred with APP/PS1dE9 mice (MMRRC strain 034832) that expressed the human Swedish mutation within the $A \beta$ domain of the amyloid precursor protein (APPK594N/M595L). Additionally, these mice carried a transgene encoding human presenilin 1 with a deletion of the exon 9 . Both transgenes were expressed under the prion protein promoter ${ }^{21}$.

\section{Brain slice preparation:}

As described in a previous publication ${ }^{22}$, mice were deeply anesthetized with an i.p. injection of ketamine/xylazine $(0.26 / 0.02 \mathrm{mg} / \mathrm{g}$ ) and transcardially perfused with PBS (pH 7.4) followed by perfusion with $4 \%$ paraformaldehyde (PFA) for five minutes each. Subsequently, the brains were removed and post fixed overnight in $4 \%$ PFA at $4^{\circ} \mathrm{C}$. Next, 50-100 $\mu \mathrm{m}$ thick brain sections were cut on a vibratome (Leica VT1200S). Un-stained slices were mounted between standard microscope slides and coverslips for SRS and CARS imaging.

\section{SRS and CARS Microscopy:}

Coherent Raman Scattering Microscopy (CARS and SRS) was performed on a Leica SP8 CARS laser scanning microscope with SRS option (Leica Microsystems, Mannheim, Germany). Supplemental Figure S1 shows a schematic of the beam routing. Briefly, CRS signals are excited using two temporally and spatially overlapped pulse trains from a PicoEmerald S optical parametric oscillator (APE, Berlin, Germany). The pump beam wavelength is fixed at $1031.25 \mathrm{~nm}$, and the Stokes beam is tunable from 720 $-980 \mathrm{~nm}$, allowing the excitation of vibrations in the range of 4200 to $500 \mathrm{~cm}^{-1}$. Both pulses are 2 picoseconds in duration, providing a $\sim 12 \mathrm{~cm}^{-1}$ spectral resolution of the total system. For SRS microscopy, the Stokes beam intensity is modulated at $20 \mathrm{MHz}$ using an Electro-Optical Modulator (EOM). To acquire SRS signals, the Pump beam intensity is recorded in the forward direction using a silicon photodiode, and demodulated using a Lock-In amplifier (Zürich Instruments, Zürich, Switzerland). CARS microscopy does not require intensity modulation, and signals are detected on photomultiplier tubes in the transmitted and epi-detected directions. Second harmonic generation (SHG) / two-photon excited fluorescence signals can be recorded simultaneously with the CARS signals. Forward SRS and EpiCARS/SHG detection are possible simultaneously. Visible-light confocal laser scanning microscopy was performed on the same instrument, and used e.g. for Methoxy-04 co-staining experiments. 


\section{Citations:}

1. Saar, B. G. et al. Video-rate molecular imaging in vivo with stimulated Raman scattering. Science 330, 1368-70 (2010).

2. Cheng, J. X. \& Xie, X. S. Vibrational spectroscopic imaging of living systems: An emerging platform for biology and medicine. Science (80-. ). 350, aaa8870-aaa8870 (2015).

3. Camp Jr, C. H. \& Cicerone, M. T. Chemically sensitive bioimaging with coherent Raman scattering. Nat. Photonics 9, 295-305 (2015).

4. Lee, H. J. et al. Label-Free Vibrational Spectroscopic Imaging of Neuronal Membrane Potential. J. Phys. Chem. Lett. 8, 1932-1936 (2017).

5. Fu, D., Yang, W. \& Xie, X. S. Label-free Imaging of Neurotransmitter Acetylcholine at Neuromuscular Junctions with Stimulated Raman Scattering. J. Am. Chem. Soc. 139, 583-586 (2017).

6. Kiskis, J. et al. Plaque-associated lipids in Alzheimer's diseased brain tissue visualized by nonlinear microscopy. Sci. Rep. 5, 1-9 (2015).

7. Ji, M. et al. Label-free imaging of amyloid plaques in Alzheimer's disease with stimulated Raman scattering microscopy. Sci. Adv. 4, eaat7715 (2018).

8. Lee, J. H., Kim, D. H., Song, W. K., Oh, M.-K. \& Ko, D.-K. Label-free imaging and quantitative chemical analysis of Alzheimer's disease brain samples with multimodal multiphoton nonlinear optical microspectroscopy. J. Biomed. Opt. 20, 56013 (2015).

9. Shahmoradian, S. H. et al. Lewy pathology in Parkinson's disease consists of crowded organelles and lipid membranes. Nat. Neurosci. 22, 1099-1109 (2019).

10. Poon, K. W. C. et al. Lipid biochemical changes detected in normal appearing white matter of chronic multiple sclerosis by spectral coherent Raman imaging. Chem. Sci. 9, 1586-1595 (2018).

11. Tian, F. et al. Monitoring peripheral nerve degeneration in ALS by label-free stimulated Raman scattering imaging. Nat. Commun. 7, 13283 (2016).

12. Cicerone, M. T. \& Camp, C. H. Histological coherent Raman imaging: a prognostic review. Analyst 143, 33-59 (2018).

13. Ji, M. et al. Rapid, Label-Free Detection of Brain Tumors with Stimulated Raman Scattering Microscopy. Sci. Transl. Med. 5, 201ra119-201ra119 (2013).

14. Ji, M. et al. Detection of human brain tumor infiltration with quantitative stimulated Raman scattering microscopy. Sci. Transl. Med. 7, 309ra163-309ra163 (2015).

15. Orringer, D. A. et al. Rapid intraoperative histology of unprocessed surgical specimens via fibrelaser-based stimulated Raman scattering microscopy. Nat. Biomed. Eng. 1, (2017).

16. Yue, S. et al. Cholesteryl ester accumulation induced by PTEN loss and PI3K/AKT activation underlies human prostate cancer aggressiveness. Cell Metab. 19, 393-406 (2014).

17. Li, J. et al. Lipid Desaturation Is a Metabolic Marker and Therapeutic Target of Ovarian Cancer Stem Cells. Cell Stem Cell 20, 303-314 (2017). 
18. Chen, T. et al. Stimulated Raman Scattering Micro-dissection Sequencing (SMD-Seq) for Morphology-specific Genomic Analysis of Oral Squamous Cell Carcinoma. bioRxiv 121616 (2017). doi:10.1101/121616

19. Tu, H. et al. Stain-free histopathology by programmable supercontinuum pulses. Nat. Photonics 10, 534-540 (2016).

20. Satoh, S. et al. Label-free visualization of acetaminophen-induced liver injury by high-speed stimulated Raman scattering spectral microscopy and multivariate image analysis. Pathol. Int. 64, 518-526 (2014).

21. Jankowsky, J. L. et al. APP processing and amyloid deposition in mice haplo-insufficient for presenilin 1. Neurobiol. Aging 25, 885-892 (2004).

22. Schmid, L. C. et al. Dysfunction of Somatostatin-Positive Interneurons Associated with Memory Deficits in an Alzheimer's Disease Model. Neuron 92, 114-125 (2016).

23. Chen, P. et al. Raman signature from brain hippocampus could aid Alzheimer's disease diagnosis. Appl. Opt. 48, 4743-8 (2009).

24. Michael, R. et al. Hyperspectral Raman imaging of neuritic plaques and neurofibrillary tangles in brain tissue from Alzheimer's disease patients. Sci. Rep. 7, 15603 (2017).

25. Wong, M. W. et al. Dysregulation of lipids in Alzheimer's disease and their role as potential biomarkers. Alzheimer's Dement. 13, 810-827 (2017).

26. Czamara, K. et al. Raman spectroscopy of lipids: A review. J. Raman Spectrosc. 46, 4-20 (2015).

27. Wu, H. et al. In vivo lipidomics using single-cell Raman spectroscopy. Proc. Natl. Acad. Sci. 108, 3809-3814 (2011).

28. Hosokawa, M. et al. In vivo live cell imaging for the quantitative monitoring of lipids by using raman microspectroscopy. Anal. Chem. 86, 8224-8230 (2014).

29. Wang, P. et al. Label-free quantitative imaging of cholesterol in intact tissues by hyperspectral stimulated Raman scattering microscopy. Angew. Chemie - Int. Ed. 52, 13042-13046 (2013).

30. Arbor, S. C., LaFontaine, M. \& Cumbay, M. Amyloid-beta Alzheimer targets - protein processing, lipid rafts, and amyloid-beta pores. Yale J. Biol. Med. 89, 5-21 (2016).

31. Praticò, D., Uryu, K., Leight, S., Trojanoswki, J. Q. \& Lee, V. M. Increased lipid peroxidation precedes amyloid plaque formation in an animal model of Alzheimer amyloidosis. J. Neurosci. 21, 4183-7 (2001).

32. Kaya, I. et al. Delineating Amyloid Plaque Associated Neuronal Sphingolipids in Transgenic Alzheimer's Disease Mice (tgArcSwe) Using MALDI Imaging Mass Spectrometry. ACS Chem. Neurosci. 8, 347-355 (2017).

33. Han, S. et al. Amyloid plaque structure and cell surface interactions of $\beta$-amyloid fibrils revealed by electron tomography. Sci. Rep. 7, 43577 (2017).

34. Martins, I. C. et al. Lipids revert inert Abeta amyloid fibrils to neurotoxic protofibrils that affect learning in mice. EMBO J. 27, 224-33 (2008).

35. Panchal, M. et al. Enrichment of cholesterol in microdissected Alzheimer's disease senile plaques 
as assessed by mass spectrometry. J. Lipid Res. 51, 598-605 (2010).

36. Torres, M., Busquets, X. \& Escriba, P. V. Brain Lipids in the Pathophysiology and Treatment of Alzheimer's Disease. Updat. Dement. 127-167 (2016). doi:10.5772/64757

37. Joshi, P. et al. Microglia convert aggregated amyloid- $\beta$ into neurotoxic forms through the shedding of microvesicles. Cell Death Differ. 21, 582-93 (2014).

38. Condello, C., Yuan, P., Schain, A. \& Grutzendler, J. Microglia constitute a barrier that prevents neurotoxic protofibrillar A 342 hotspots around plaques. Nature Communications 6, (2015).

39. Gyonevaa, S., Swangera, S. A., Zhanga, J., Weinshenkerb, D. \& Traynelis, S. F. Altered motility of plaque-associated microglia in a model of Alzheimer's disease. Neuroscience 330, 410-420 (2016).

40. Baker, M. J. et al. Clinical applications of infrared and Raman spectroscopy: state of play and future challenges. Analyst 143, 1735-1757 (2018).

41. Pence, I. \& Mahadevan-Jansen, A. Clinical instrumentation and applications of Raman spectroscopy. Chem. Soc. Rev. 45, 1958-79 (2016). 